\title{
Efeito das estações do ano no pico de fluxo expiratório de idosos institucionalizados e não institucionalizados
}

\author{
Effect of the seasons on the peak expiratory flow in institutionalized and noninstitutionalized \\ elderly
}
Efecto de las estaciones del año en el flujo espiratorio máximo de ancianos institucionalizados y no institucionalizados

Mateus Dias Antunes', Sthefany Dlugosz Silva², Braulio Henrique Magnani Branco ${ }^{3}$, Fernanda Shizue Nishida4, Amélia Pasqual Marques ${ }^{5}$, Sonia Maria Marques Gomes Bertolini ${ }^{6}$

RESUMO I As doenças respiratórias afetam milhões de pessoas, principalmente os idosos, e as mudanças climáticas estão entre os fatores predisponentes, interferindo na saúde dessa população. O objetivo deste estudo foi avaliar o pico de fluxo expiratório de idosos institucionalizados e não institucionalizados durante as quatro estações do ano. Estudo de coorte prospectivo com 67 idosos de ambos os sexos, residentes na cidade de Maringá (PR) e divididos em dois grupos: idosos institucionalizados ( $n=37$ ) e idosos não institucionalizados $(n=30)$. Os dados foram coletados durante um mês, uma vez por semana nas quatro estações do ano, totalizando 16 avaliações. O pico de fluxo expiratório foi avaliado com o equipamento peak flow meter. A comparação dos dois grupos de idosos foi feita por análise de variância de dois fatores utilizando o post-hoc de Bonferroni. A menor média de pico de fluxo expiratório para os idosos institucionalizados e não institucionalizados foi no verão $(176,2 \pm 60,2$ e $263,2 \pm 116,2)$, seguido pelo outono $(193,4 \pm 59,5$ e $287,5 \pm 118)$, inverno $(215,3 \pm 82,5$ e $291,5 \pm 08,4)$ e primavera $(221,7 \pm 83,5$ e $291,5 \pm 08,4)$. Conclui-se que 0 pico de fluxo expiratório de idosos varia de acordo com as estações do ano, porém os institucionalizados apresentam valores mais baixos. Os mais altos são encontrados na primavera, embora aquém do valor predito para os idosos de ambos os grupos.

Descritores | Envelhecimento; Mudança Climática; Promoção da Saúde.

\begin{abstract}
I Respiratory diseases affect millions of people, especially the elderly, and climate change is among the predisposing factors interfering with the health of this population. This study aimed to evaluate the peak expiratory flow in institutionalized and noninstitutionalized elderly during the four seasons of the year. A prospective cohort study with 67 elderly men and women living in the city of Maringá, Paraná, Brazil, divided into two groups: institutionalized elderly ( $n=37)$ and noninstitutionalized elderly $(n=30)$. The data were collected for one month, once a week in the four seasons of the year, totaling 16 evaluations. The peak expiratory flow was evaluated using the Peak-Flow Meter equipment. The two groups of elderly were compared by two-way analysis of variance using the Bonferroni post-hoc. The lowest mean peak expiratory flow for institutionalized and noninstitutionalized elderly was observed in the summer (176.2 \pm 60.2 and $263.2 \pm 116.2$ ), followed by fall (193.4 \pm 59.5 and $287.5 \pm 118)$, winter $(215.3 \pm 82.5$ and $291.5 \pm 08.4)$, and spring (221.7 \pm 83.5 and $291.5 \pm 08.4)$.
\end{abstract}

Pesquisa realizada no Centro Universitário de Maringá (Unicesumar) - Maringá (PR), Brasil.

Universidade de São Paulo (USP) - São Paulo (SP), Brasil. E-mail: mateusantunes@usp.br. Orcid: 0000-0002-2325-2548

${ }^{2}$ Centro Universitário de Maringá (Unicesumar) - Maringá (PR), Brasil. E-mail: stefany_tuty@hotmail.com. Orcid: 0000-0001-6789-0819

${ }^{3}$ Centro Universitário de Maringá (Unicesumar) - Maringá (PR), Brasil. E-mail: braulio.branco@unicesumar.edu.br. Orcid:0000-0002-4625-9128

${ }^{4}$ Centro Universitário de Maringá (Unicesumar)-Maringá(PR),Brasil.E-mail:fernanda.nishida@unicesumar.edu.br.Orcid:0000-0002-5078-9017

${ }^{5}$ Universidade de São Paulo (USP) - São Paulo (SP), Brasil. E-mail: pasqual@usp.br. Orcid: 0000-0001-6705-7763

${ }^{6}$ Centro Universitário de Maringá (Unicesumar) - Maringá (PR), Brasil. E-mail: sonia.bertolini@unicesumar.edu.br. Orcid: 0000-0003-2579-7362 
The conclusion was that the peak of expiratory flow of the elderly varies according to the seasons, but the institutionalized ones have lower values. The highest values are found in the spring, although below the value predicted for the elderly of both groups.

Keywords | Aging; Climate Change; Health Promotion.

RESUMEN | Las enfermedades respiratorias afectan a millones de personas, especialmente a los ancianos, y el cambio climático es uno de los factores predisponentes que interfieren en la salud de esta población. El presente estudio tuvo como objetivo evaluar el flujo espiratorio máximo de ancianos institucionalizados y no institucionalizados durante las cuatro estaciones del año. Se realizó un estudio prospectivo de cohorte con 67 ancianos de ambos sexos que viven en la ciudad de Maringá (PR), los cuales se dividieron en dos grupos: ancianos institucionalizados ( $n=37)$ y ancianos no institucionalizados ( $n=30)$. Los datos se recolectaron durante un mes, una vez a la semana en las cuatro estaciones del año, y totalizó 16 evaluaciones. El flujo espiratorio máximo se evaluó con la herramienta peak flow meter. La comparación de los dos grupos de ancianos se realizó mediante el análisis de la varianza de dos factores utilizando el post hoc de Bonferroni. El promedio más bajo del flujo espiratorio máximo para los ancianos institucionalizados y no

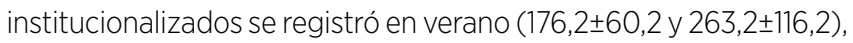
seguido del otoño $(193,4 \pm 59,5$ y $287,5 \pm 118)$, invierno $(215,3 \pm 82,5$ y $291,5 \pm 08,4)$ y primavera $(221,7 \pm 83,5$ y $291,5 \pm 08,4)$. Se concluye que el flujo espiratorio máximo de los ancianos varía según las estaciones del año, sin embargo, los ancianos institucionalizados tienen los valores más bajos. Los más altos se encuentran en la primavera, aunque por debajo del valor previsto para los ancianos de ambos grupos.

Palabras clave I Envejecimiento; Cambio Climático; Promoción de la Salud.

\section{INTRODUÇÃO}

As doenças respiratórias afetam milhões de pessoas, principalmente os idosos, sendo umas das causas mais frequentes de óbitos e adoecimentos, representando $16 \%$ das internações, tanto por fatores crônicos quanto agudos, e $30 \%$ das causas de morte, sendo que as mudanças climáticas estão entre os fatores predisponentes, interferindo no quadro saúde-doença, tanto de forma direta quanto indireta, principalmente em afecções nas vias aéreas ${ }^{1}$.

As vias aéreas recebem influência da exposição a poluentes das condições ambientais (poeira, fungos e má higiene), do tabagismo, da baixa condição socioeconômica, da exposição a agentes biológicos e da sazonalidade climática ${ }^{2}$. No decorrer do ano, o clima no Brasil apresenta variações, dentre elas, períodos intensos de seca ou chuvas abundantes ${ }^{3}$. As alterações climáticas estão relacionadas com diversos fatores e sofrem influência da atmosfera, camada de ozônio, industrialização, urbanização, carros e lixo $^{4}$; contribuindo para que o ar se torne mais prejudicial à saúde da população, com efeitos significativos sobre as condições respiratórias e cardíacas ${ }^{3}$.

A alta concentração de poluição no ar é um forte indício para as doenças respiratórias, cardiovasculares, neurológicas e diversos tipos de câncer ${ }^{5}$. Longos períodos, tanto de chuva quanto de seca, influenciam essas condições, uma vez que, em períodos mais secos, há maior exposição à poeira, facilmente irritativas às vias respiratórias e, em dias mais chuvosos e úmidos, as doenças respiratórias se tornam mais propícias, podendo, então, representar fortes agravantes a saúde. Nesse sentido, torna-se relevante ficar atento aos parâmetros respiratórios em diferentes épocas do ano ${ }^{6}$.

Um importante parâmetro é o pico de fluxo expiratório (PFE), considerado como fluxo máximo alcançado durante uma manobra expiratória forçada, partindo do volume pulmonar máximo (capacidade vital). É um parâmetro que serve para definir a presença ou ausência de obstrução das vias aéreas, quantificar o grau de estreitamento e obstrução dos brônquios, avaliar a eficácia da tosse e a capacidade de resposta ao uso de broncodilatadores ${ }^{7}$. $\mathrm{O}$ PFE é um equipamento de baixo custo, fácil aplicação e não invasivo ${ }^{8}$.

Apesar da relação do meio ambiente e saúde estarem claras, quase todas as análises são retrospectivas por meio de base de dados e internações hospitalares. Nesse sentido, faz-se necessário, portanto, desenvolver estudos que verifiquem a associação entre as estações do ano e o PFE em idosos institucionalizados e não institucionalizados, com vistas à criação de ações de promoção da saúde do idoso, com foco no ambiente no contexto da interdisciplinaridade. Este estudo teve como hipótese que os idosos institucionalizados apresentam um $\mathrm{PFE}$ menor que os não institucionalizados. $\mathrm{O}$ objetivo deste estudo foi analisar o efeito das estações do ano no pico expiratório máximo de idosos institucionalizados e não institucionalizados. 


\section{METODOLOGIA}

Trata-se de um estudo de coorte prospectivo, com idosos institucionalizados e não institucionalizados da cidade de Maringá (PR), sendo os dados coletados entre os meses de janeiro e novembro de 2017, durante o período de um mês, uma vez por semana nas quatro estações do ano, totalizando 16 avaliações. Foi aprovado pelo Comitê de Ética do Centro Universitário de Maringá, sob o parecer número 1.911.479. Todos os participantes assinaram o termo de consentimento livre e esclarecido.

Os critérios de inclusão eram idosos, de ambos os sexos, com idade $\geq 60$ anos, residentes em instituição de longa permanência para idosos (ILPI) ou idosos não institucionalizados cadastrados em uma Unidade Básica de Saúde de Maringá (PR). Como critérios de exclusão, foram considerados os idosos que apresentaram diagnóstico clínico de doenças respiratórias obstrutivas crônicas e os cardiopatas descompensados, assim como os idosos com demências, os acamados e usuários de cadeiras de rodas. Foram excluídos do estudo os idosos que, no momento da entrevista, não fossem capazes de compreender as instruções devido a problemas cognitivos, avaliados por meio do miniexame do estado mental $(\mathrm{MEM})^{9}$, considerado um instrumento de fácil aplicação e de boa confiabilidade.

A amostra foi selecionada por conveniência para facilitar a coleta de dados dos idosos não institucionalizados que residiam no mesmo bairro que a ILPI. Inicialmente totalizou com 105 idosos, sendo 55 idosos de uma ILPI e 50 não institucionalizados, moradores do mesmo bairro da referida instituição. Ao decorrer do longo estudo, tiveram algumas perdas, portanto, 67 idosos participaram deste estudo, sendo 37 no grupo institucionalizado e 30 no grupo não institucionalizado, representado na Figura 1.

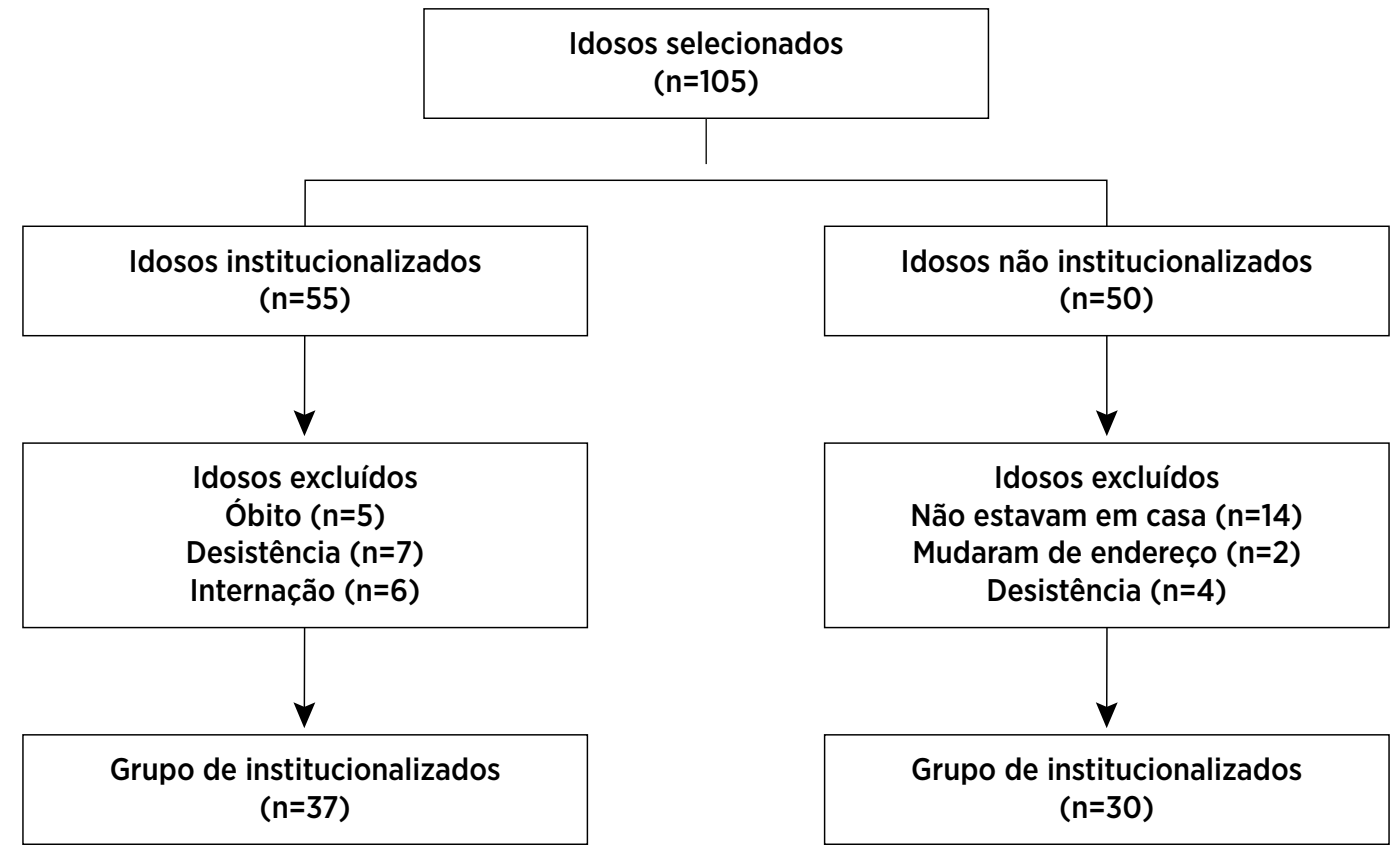

Figura 1. Fluxograma de participação dos idosos no estudo

O PFE foi avaliado com o equipamento peak flow meter, da marca Medicate, registrado pela Agência Nacional de Vigilância Sanitária (Anvisa) sob o número 10332170038, de alta confiabilidade, baixo custo, portátil, de material plástico, contendo um sistema graduado de medidas que avalia a força e a velocidade de saúde de ar dentro dos pulmões em litros por minuto $(\mathrm{L} / \mathrm{min})$. Para realizar o teste, todos os idosos foram orientados a sentarem-se confortavelmente, com os pés apoiados no chão e, posteriormente, foi solicitado que realizassem uma inspiração máxima e em seguida expirassem forçadamente e rapidamente no bocal do aparelho. Diante disso, os pesquisadores observaram com cautela qualquer escape de ar, a fim de evitar interferência nas medidas. O teste foi realizado três vezes e foi calculada a média dos resultados para identificar as possíveis diferenças nas comparações intergrupos. Para analisar os resultados, foram utilizados os valores preditos, de acordo com o sexo, idade e estatura ${ }^{10}$.

Os dados foram coletados semanalmente durante quatro semanas sempre às quartas-feiras, no período da 
tarde, totalizando 16 avaliações no decorrer das quatro estações do ano com início no verão (18 de janeiro a 8 de fevereiro), depois no outono (19 de abril a 10 de maio), no inverno (19 de julho a 9 de agosto) e na primavera (25 de outubro a 15 de novembro).

A estatística descritiva baseou-se no cálculo da média e desvio padrão dos dados coletados, após a confirmação da normalidade por meio do teste de Kolmogorov-Smirnov. Dessa forma, para comparar os dois grupos de idosos (institucionalizados e não institucionalizados), empregouse uma análise de variância a dois fatores (Anova): (grupo x estação), utilizando o post-hoc de Bonferroni caso fosse encontrada diferença significativa. Para a Anova, utilizouse o teste de Mauchly para testar a esfericidade, bem como a correção de Greenhouse-Geisser, se necessário. Foi adotado um nível de significância de 5\% para as análises que foram realizadas por meio do programa Statistica 12.0 (StatSoft, Inc., Tulsa, Estados Unidos da América).

\section{RESULTADOS}

A amostra final totalizou com 67 idosos, sendo $37(55,2 \%)$ institucionalizados e $30(44,8 \%)$ não institucionalizados. A média da idade foi de $73,6 \pm 7,42$ e $69,8 \pm 7,88$ anos para idosos institucionalizados e não institucionalizados, respectivamente (Tabela 1 ).

Tabela 1. Dados sociodemográficos de idosos institucionalizados e não institucionalizados no município de Maringá (PR)

\begin{tabular}{|c|c|c|}
\hline $\begin{array}{l}\text { Dados } \\
\text { sociodemográficos }\end{array}$ & $\begin{array}{c}\text { Idosos } \\
\text { institucionalizados } \\
(n=37 / \%)\end{array}$ & $\begin{array}{c}\text { Idosos não } \\
\text { institucionalizados } \\
(n=30 / \%)\end{array}$ \\
\hline \multicolumn{3}{|l|}{ Sexo } \\
\hline Masculino & $14(37,8 \%)$ & $7(23,3 \%)$ \\
\hline Feminino & $23(62,2 \%)$ & $23(76,7 \%)$ \\
\hline \multicolumn{3}{|l|}{ Idade (anos) } \\
\hline $60-69$ & $11(29,7 \%)$ & $17(56,7 \%)$ \\
\hline $70-79$ & $18(48,6 \%)$ & $8(26,6 \%)$ \\
\hline $80-89$ & $8(21,7 \%)$ & $5(16,7 \%)$ \\
\hline \multicolumn{3}{|l|}{ Tabagismo } \\
\hline Sim & $7(18,9 \%)$ & $4(13,3 \%)$ \\
\hline Não & $30(81,1 \%)$ & $26(86,7 \%)$ \\
\hline
\end{tabular}

A Tabela 2 apresenta o PFE nas diferentes estações do ano. O valor predito para o grupo de idosos institucionalizados foi de $453,8 \pm 41,8$ e para os não institucionalizados foi $425,5 \pm 37,9$. O verão e o outono foram as estações que apresentaram menores valores, porém com diferença estatisticamente significante entre os grupos.
Tabela 2. Média, desvio padrão e valor p das comparações entre o pico de fluxo expiratório em diferentes estações

$\begin{array}{lccc}\begin{array}{l}\text { Estações do } \\ \text { ano }\end{array} & \begin{array}{c}\text { Idosos } \\ \text { institucionalizados } \\ (\mathbf{n}=37)\end{array} & \begin{array}{c}\text { Idosos não } \\ \text { institucionalizados } \\ (\mathbf{n}=30)\end{array} & p \\ \text { Verão } & 176,2 \pm 60,2^{\text {a }} & 263,2 \pm 116,2^{\text {a }} & <0,01^{*} \\ \text { Outono } & 193,4 \pm 59,5^{\mathrm{b}} & 287,5 \pm 118,8^{\mathrm{b}} & <0,01^{*} \\ \text { Inverno } & 215,3 \pm 82,5 & 291,5 \pm 08,4 & 0,05 \\ \text { Primavera } & 221,7 \pm 83,5 & 295,4 \pm 115,2 & 0,05\end{array}$

a: valores inferiores para o verão quando comparado às demais estações do ano para ambos os grupos; b: valores inferiores para o outono quando comparado à primavera para ambos os grupos. *Diferença significativa $(p<0,05)$.

$\mathrm{Na}$ Tabela 3, verifica-se a comparação entre a média do PFE nas diferentes semanas das estações do ano, em ambos os grupos. Como pode ser observado neste estudo, o PFE apresentou valores diferentes em todas as estações do ano em ambos os grupos, embora as diferenças tenham sido significativas apenas no verão em relação às demais estações e no outono em relação à primavera e ao verão.

Tabela 3. Comparações do pico de fluxo expiratório entre as diferentes semanas das quatro estações do ano

$\begin{array}{lccc}\begin{array}{l}\text { Estações do } \\ \text { ano }\end{array} & \begin{array}{c}\text { Idosos } \\ \text { institucionalizados } \\ (\mathbf{n}=37)\end{array} & \begin{array}{c}\text { Idosos não } \\ \text { institucionalizados } \\ (\mathbf{n}=30)\end{array} & \mathbf{p} \\ \text { Verão 1 } & 163,2 \pm 58,8^{\mathrm{a}} & 261,7 \pm 113,5^{\mathrm{a}} & 0,01^{*} \\ \text { Verão 2 } & 177,8 \pm 64,3^{\mathrm{a}} & 269,1 \pm 117,8^{\mathrm{a}} & 0,01^{*} \\ \text { Verão 3 } & 175,5 \pm 63,1^{\mathrm{a}} & 249,9 \pm 117,3^{\mathrm{a}} & 0,01^{*} \\ \text { Verão 4 } & 188,5 \pm 65,4^{\mathrm{a}} & 272,0 \pm 128,0^{\mathrm{a}} & 0,01^{*} \\ \text { Outono 1 } & 176,5 \pm 60,5^{\mathrm{b}} & 274,3 \pm 122,4^{\mathrm{b}} & 0,01^{*} \\ \text { Outono 2 } & 195,0 \pm 61,8^{\mathrm{c}} & 293,1 \pm 121,7^{\mathrm{c}} & 0,01^{*} \\ \text { Outono 3 } & 198,6 \pm 65,5^{\mathrm{d}} & 287,2 \pm 122,1^{\mathrm{d}} & 0,01^{*} \\ \text { Outono 4 } & 203,5 \pm 65,9^{\mathrm{e}} & 295,6 \pm 118,1^{\mathrm{e}} & 0,01^{*} \\ \text { Inverno 1 } & 189,8 \pm 75,9^{\mathrm{f}} & 288,6 \pm 111,8^{\mathrm{f}} & 0,01^{*} \\ \text { Inverno 2 } & 215,2 \pm 87,2^{\mathrm{a}} & 287,2 \pm 107,1^{\mathrm{a}} & 0,01^{*} \\ \text { Inverno 3 } & 220,4 \pm 59,5 & 288,1 \pm 107,0 & 0,05 \\ \text { Inverno 4 } & 235,8 \pm 91,8 & 302,0 \pm 119,3 & 0,05 \\ \text { Primavera 1 } & 196,0 \pm 76,3 & 292,6 \pm 115,2 & 0,05 \\ \text { Primavera 2 } & 222,1 \pm 90,3 & 290,6 \pm 115,2 & 0,05 \\ \text { Primavera 3 } & 226,6 \pm 88,3 & 293,4 \pm 115,0 & 0,05 \\ \text { Primavera 4 } & 242,0 \pm 92,7^{\mathrm{h}} & 305,1 \pm 115,9^{\mathrm{h}} & 0,01^{*}\end{array}$

a: valores inferiores quando comparado a todas as semanas de outono, inverno e primavera; $b$ : valores inferiores para quando comparado ao outono 4, inverno 2, 3 e 4 e primavera 2, 3 e 4; c: valores inferiores quando comparado ao inverno 4 e primavera 4 ; $d$ : maiores valores quando comparado ao verão 1 e 3 e menores valores quando comparado ao inverno 4 e primavera 4; e: maiores valores quando comparado ao outono 1 e menores valores quando comparado à primavera 4; f: menores valores quando comparado ao inverno 4 e primavera 4; g: menores valores quando comparado ao inverno 4 ; $h$ : maiores valores quando comparado à primavera 1. *Diferença significativa $(p<0,05)$. 
Quando se comparou a primavera, estação com melhores escores, com o valor predito, a diferença foi altamente significativa $(\mathrm{p}<0,001)$ para ambos os grupos (Tabela 4).

Tabela 4. Média, desvio padrão e valor de p do grupo institucionalizado e não institucionalizado referente à primavera e ao valor predito

\begin{tabular}{|c|c|c|c|}
\hline $\begin{array}{l}\text { Estações do } \\
\text { ano }\end{array}$ & $\begin{array}{c}\text { Idosos } \\
\text { institucionalizados } \\
(n=37)\end{array}$ & $\begin{array}{c}\text { Idosos não } \\
\text { institucionalizados } \\
(n=30)\end{array}$ & p \\
\hline PFE obtido & $221,7 \pm 83,5$ & $295,4 \pm 115,2$ & $0,001^{*}$ \\
\hline PFE predito & $453,83 \pm 41,85$ & $425,53 \pm 37,91$ & $0,001^{*}$ \\
\hline
\end{tabular}

PFE: Pico de fluxo expiratório. *Diferença significativa $(p<0,05)$.

\section{DISCUSSÃO}

Nosso estudo teve como objetivo analisar o efeito das estações do ano no PFE de idosos institucionalizados e não institucionalizados, e nossos resultados indicam que o PFE de idosos varia de acordo com as estações do ano, sendo que os melhores valores são encontrados na primavera, embora aquém do valor predito para os idosos.

Neste estudo, o verão foi a estação do ano com menor $\mathrm{PFE}$ em ambos os grupos. A elevação da temperatura afeta o sistema respiratório, sendo o verão o período de maior risco para desenvolvimento de doenças inflamatórias ou infecciosas. No verão, há uma diminuição no período de chuva, ocasionando uma modificação da temperatura e umidade do ar, que contribui para problemas de saúde ${ }^{11}$. O índice de mortalidade induzida pelo calor é de 5,33\%, com maior risco para pneumonia ${ }^{12}$.

Como esperado, as temperaturas do verão têm aumentado, em média, a uma taxa de $0,32^{\circ} \mathrm{C}$ por década $^{13} . \mathrm{Na}$ Espanha, a vulnerabilidade associada ao calor diminuiu para a faixa de temperaturas extremas do verão, sugerindo uma resposta de ajuste da sociedade espanhola ao aumento das temperaturas, apesar do envelhecimento da população ${ }^{14}$. Outro fator potencial que contribui para a redução dos riscos de mortalidade pode ter sido o plano nacional de ações preventivas contra os efeitos do excesso de temperatura na saúde do Ministério da Saúde da Espanha ${ }^{15}$, que foi implementado em 2004. No Brasil, os dados de mortalidade relacionados ao calor aumentam, no entanto, em 2016 foi criado o plano nacional de adaptação à mudança do clima ${ }^{16}$, que busca promover a gestão e redução do risco associado à mudança do clima.

Recentemente, um estudo ${ }^{17}$ sobre o impacto que as temperaturas extremas têm na saúde das pessoas analisou vinte países em quatro continentes e prevê que o aumento na mortalidade no frio nos próximos anos (2031 a 2080) deve ser maior próximo à linha do Equador, e o Brasil, as Filipinas e a Colômbia serão os países mais afetados, enquanto os Estados Unidos e a Europa serão as regiões menos afetadas. Esses resultados evidenciam que as informações atualmente disponíveis sobre climas futuros são suficientes para orientar o planejamento regional na saúde pública ${ }^{18}$.

A umidade é um dos contribuintes para a sensação de conforto e bem estar relacionado à saúde dos indivíduos, sendo que, com as mudanças climáticas e redução de 30\% da umidade do ar, o organismo acaba sendo submetido a alterações para manutenção da temperatura corporal de acordo com a temperatura ambiente ${ }^{19}$. Essas alterações agridem o sistema imunológico, tornando os indivíduos mais suscetíveis a complicações respiratórias, ressecamento de mucosas e sangramentos nasais ${ }^{20}$.

A população idosa é mais vulnerável às mudanças de temperatura. $\mathrm{O}$ estresse causado pelo calor faz com que o sistema imunológico não reaja tão bem a essa mudança de temperatura, levando a complicações do organismo. A exposição às variações climáticas torna os longevos mais suscetíveis, principalmente a problemas respiratórios, tendo como severidade neste período de elevação térmica o agravamento de bronquite crônica e pneumonia ${ }^{21}$.

Os maiores valores de PFE foram encontrados na primavera, seguida do inverno, contudo, inferiores aos valores preditos para os idosos. Como mostrado no estudo de Ruivo et al. ${ }^{22}$, existem diferenças do padrão respiratório entre jovens adultos e idosos saudáveis, sugerindo que a função pulmonar é influenciada pelo envelhecimento cronológico.

Apesar do inverno ter sido a segunda estação com melhores resultados no PFE, nessa estação do ano também ocorre maior agravamento do comprometimento respiratório e incapacidade funcional, devido à diminuição da umidade e temperatura, tornando o trato respiratório mais exposto a alterações, ocasionando hiper-reatividade brônquica, que induz a inflamação crônica e aumenta os sintomas respiratórios ${ }^{23}$.

A diminuição da umidade relativa do ar com valores inferiores a 30\% é considerada um risco para a integridade das vias aéreas, dificultando a homeostase interna do sistema respiratório. Nos meses chuvosos, em contraposição à problemática vivenciada nos meses de seca, a alta umidade relativa do ar, aliada ao maior tempo de permanência nos ambientes internos, ao menor arejamento e exposição ao sol dos espaços domiciliares, por 
consequência, favorece o crescimento de mofo e fungos, e esses são fatores que podem contribuir para o aumento das doenças respiratórias, especialmente as alérgicas ${ }^{24}$. Além do mais, alguns vírus apresentam comportamento altamente sazonal, com maior frequência no período frio em regiões de clima temperado e também no período de chuvas nas de clima tropical. Esses vírus são causadores frequentes de infecções respiratórias, principalmente das vias aéreas superiores ${ }^{25}$.

Em um estudo realizado por Antunes et al. ${ }^{26}$, que avaliaram e compararam o PFE entre idosos institucionalizados e não institucionalizados, verificou-se que os valores de PFE foram significativamente maiores nos idosos não institucionalizados. Neste estudo, os valores do PFE também são menores nos idosos institucionalizados, comparado aos não institucionalizados.

Nesse sentido, a realização de testes pulmonares por fisioterapeutas auxilia os profissionais da saúde na detecção de condições patológicas ${ }^{26,27}$, bem como na avaliação da função de órgãos e sistemas corpóreos ${ }^{28,29}$. Faz-se necessário criar novas estratégias e ações que promovam a saúde dos idosos institucionalizados e não institucionalizados no contexto da interdisciplinaridade.

Um ponto favorável desta investigação foi o período da coleta de dados, assim como ter coletado dados de idosos circunscritos na mesma região geográfica e caracterizados com bom estado mental. Este estudo mostra a importância da atuação do fisioterapeuta na realização de testes visando ao acompanhamento da saúde do idoso tanto na comunidade quanto em ILPI.

Algumas limitações merecem ser destacadas neste estudo: além do tamanho e tipo de amostragem, destaca-se o fato de não terem sidos coletados dados sobre parâmetros meteorológicos como pressão atmosférica, temperatura e umidade relativa do ar, precipitação, radiação solar e direção e velocidade do vento, bem como a falta de conhecimento da prática de atividade física dos idosos, que poderia influenciar nos resultados. Além disso, ao decorrer do estudo, outras limitações foram o comprometimento do idoso a participar durante o ano das coletas, pois os dados tinham que ser coletados todos no mesmo dia e muitos idosos não estavam em sua residência para receber o pesquisador na data e horário agendado para avaliação.

\section{CONCLUSÃO}

Conclui-se que o pico de fluxo expiratório de idosos varia de acordo com as estações do ano e possui comportamento semelhante independentemente do grupo, sendo que os melhores valores são encontrados na primavera, embora aquém do valor predito para os idosos.

\section{REFERÊNCIAS}

1. Cruz DM, Ohara DG, Castro SS, Jamami M. Internações hospitalares, óbitos, custos com doenças respiratórias e sua relação com alterações climáticas no município de São Carlos SP, Brasil. Medicina - Rib Preto. 2016;49(3):248-57. doi: 10.11606/ issn.2176-7262.v49i3p248-257

2. Rosa AM, Ignotti E, Hacon SDS, Castro HA. Analysis of hospitalizations for respiratory diseases in Tangará da Serra, Brazil. J Bras Pneumol. 2008;34(8):575-82. doi: 10.1590/ S1806-37132008000800006

3. Ribeiro H, Silva EN, Oliveira MA, Oliver SL. Ritmo climático e geografia da saúde. Rev Geogr. 2016 [cited 2019 Jul 5];33(4):123. Available from: https://periodicos.ufpe.br/revistas/ revistageografia/article/view/229307

4. Mendonça FA, Castelhano FJ. O clima e a poluição do ar por PTS em Curitiba - PR. RDG. 2016;(spe):133-44. doi: 10.11606/ rdg.vOispe.120677

5. Nascimento AP, Santos JM, Mill JG, Souza JB, Reis Júnior NC, Reisen VA. Association between the concentration of fine particles in the atmosphere and acute respiratory diseases in children. Rev Saúde Pública. 2017;51(3):1-10. doi: 10.1590/ s1518-8787.2017051006523

6. Confalonieri UEC. Mudança climática global e saúde humana no Brasil. Parcerias estratégicas. 2008 [cited 2019 Jul 5];13(27):323-50. Available from: http://seer.cgee.org.br/ index.php/parcerias_estrategicas/article/viewFile/333/327

7. Presto B, Presto LDDN. Fisioterapia respiratória: uma nova visão. 3rd ed. Rio de Janeiro: Bruno Presto; 2007.

8. Silva JA, Fonseca MR, Melo MAV, Melo PM. O peak flow expiratório em mulheres fumantes e não fumantes e suas medidas de confiabilidade. Assobrafir Ciênc. 2015 [cited 2019 Jul 5];6(1):41-8. Available from: http://www.uel.br/revistas/uel/ index.php/rebrafis/article/view/19059/16189

9. Brucki SMD, Nitrini R, Caramelli P, Bertolucci PHF, Okamoto IH. Sugestões para o uso do mini-exame do estado mental no Brasil. Arq Neuropsiquiatr. 2003;61(3):777-81. doi: 10.1590/ S0004-282X2003000500014

10. Leiner GC, Abramowitz S, Small MJ, Stenby VB, Lewis WA. Expiratory peak flow rate 1: standard values for normal subjects. use as a clinical test of ventilatory function. Am Rev Respir Dis. 1963;88(5):644-51. doi: 10.1164/arrd.1963.88.5.644

11. Sousa TCM, Amancio F, Hacon SS, Barcellos C. Doenças sensíveis ao clima no Brasil e no mundo: revisão sistemática. Rev Panam Salud Pública. 2018;42:1-10. doi: 10.26633/RPSP.2018.85

12. Bunker A, Wildenhain J, Vandenbergh A, Henschke N, Rocklöv J, Hajat S, et al. Effects of air temperature on climatesensitive mortality and morbidity outcomes in the elderly: a systematic review and meta-analysis of epidemiological evidence. EBioMedicine. 2016;6(1):258-68. doi: 10.1016/j. ebiom.2016.02.034 
13. Gasparrini A, Guo Y, Hashizume M, Kinney PL, Petkova EP, Lavigne E, et al. Temporal variation in heat-mortality associations: a multicountry study. Env Health Perspect. 2015;123(11):1200-7. doi: 10.1289/ehp.1409070

14. Achebak H, Devolder D, Ballester J. Heat-related mortality trends under recent climate warming in Spain: a 36-year observational study. PLoS Med. 2018;15(7):e1002617. doi: 10.1371/ journal.pmed.1002617

15. Ministerio de Sanidad, Consumo y Bienestar Social (ES). Plan nacional de actuaciones preventivas por altas temperaturas: 2018. Madrid; 2018 [cited 2018 Sep 13]. Available from: https://www.mscbs. gob.es/ciudadanos/saludAmbLaboral/planAltasTemp/2018/docs/ Plan_Nacional_de_Exceso_de_Temperaturas_2018.pdf

16. Brasil. Ministério do Meio Ambiente. Plano nacional de adaptação à mudança do clima: 2016. Brasilia, DF; 2016 [cited 2018 Sep 13]. Available from: http://www.mma.gov.br/clima/adaptacao/ plano-nacional-de-adaptacao

17. Guo Y, Gasparrini A, Li S, Sera F, Vicedo-Cabrera AM, Coelho MSZS, et al. Quantifying excess deaths related to heatwaves under climate change scenarios: a multicountry time series modelling study. PLoS Med. 2018;15(7):e1002629. doi: 10.1371/ journal.pmed.1002629

18. Limaye VS, Vargo J, Harkey M, Holloway T, Patz JA. Climate change and heat-related excess mortality in the Eastern USA. Ecohealth. 2018;15(3):485-96. doi: 10.1007/s10393-018-1363-0

19. Azevedo JVV, Santos CAC, Silva MT, Olinda RA, Santos DAS. Análise das variações climáticas na ocorrência de doenças respiratórias por influenza em idosos na região metropolitana de João Pessoa - PB. Soc Nat. 2017;29(1):123-35. doi: 10.14393/SN-v29n1-2017-8

20. Miranda MJ. Análise temporal das internações por gripe e pneumonia associadas às variáveis meteorológicas no Município de São Paulo, SP. Rev Inst Geol. 2016;37(2):61-71. doi: 10.5935/0100-929X.20160009

21. Moreira M, Monteiro A. Agravamento da saúde e vulnerabilidades climáticas e socioeconómicas: indivíduos com AVC, dispneia e asma e dor torácica (Porto, 2005-2008). Territorium. 2016:(23):23-41. doi: 10.14195/1647-7723_23_3

22. Ruivo S, Viana P, Martins C, Baeta $C$. Effects of aging on lung function: a comparison of lung function in healthy adults and the elderly. Rev Port Pneumol. 2009;15(4):629-53. doi: 10.1016/ S0873-2159(15)30161-6

23. Hyrkäs-Palmu H, Ikäheimo TM, Laatikainen T, Jousilahti P, Jaakkola MS, Jaakkola JJ. Cold weather increases respiratory symptoms and functional disability especially among patients with asthma and allergic rhinitis. Sci Rep. 2018;8(1):10131. doi: 10.1038/s41598-018-28466-y

24. Environmental Protection Agency (US). Una breve guía para el moho la humedad y su hogar. Washington, DC; 2008 [cited 2018 Sep 13]. Available from: https://espanol.epa.gov/sites/ production-es/files/2015-08/documents/moldguide_sp_1.pdf

25. Thomazelli LM, Vieira S, Leal AL, Sousa TS, Oliveira DBL, Golono MA, et al. Vigilância de oito vírus respiratórios em amostras clínicas de pacientes pediátricos no sudeste do Brasil. J Pediatr. 2007;83(5):422-8. doi: 10.1590/S0021-75572007000600005

26. Antunes MD, Bertolini SMMG, Nishida FS. Avaliação do pico de fluxo expiratório em idosos institucionalizados e não institucionalizados. Temas Saúde. 2018 [cited 2019 Jul 5];18(2):186-203. Available from: http://temasemsaude. com/wp-content/uploads/2018/07/18212.pdf

27. Almeida AP, Cruz ICF. Patient diagnosed with impaired gas exchange: systematized literature review. J Spec Nurs Care. 2017 [cited 2019 Jul 5];9(1):1-11. Available from: http://www. jsncare.uff.br/index.php/jsncare/article/view/2906/726

28. Porto EDO, Latuf MO. Relações entre o comportamento climático e doenças respiratórias em Barreiras/BA. Geografia. 2017;26(2):164-81. doi: 10.5433/2447-1747.2017v26n2p164

29. Caldeira JB, Lima Junior NA, Sancho AG, Rosa JLS, Faria D, Balthazar MC. Avaliação do pico de fluxo expiratório em idosos autônomos institucionalizados e não institucionalizados. Fisioter Bras. 2012;13(4):272-6. doi: 10.33233/fb.v13i4.549 